\title{
Data repeatability and acquisition techniques for time-domain spectral induced polarization
}

\author{
A. Gazoty ${ }^{1}$, G. Fiandaca ${ }^{1,2}$, J. Pedersen ${ }^{1}$, E. Auken ${ }^{1}$ and A.V. Christiansen ${ }^{1}$ \\ ${ }^{1}$ HydroGeophysics Group, Department of Geoscience, Aarhus University, Denmark \\ ${ }^{2}$ Department of Mathematics and Computer Science, University of Palermo, Italy
}

Received October 2011, revision accepted January 2013

\begin{abstract}
The Time Domain Induced Polarization (TDIP) technique is widely used in applied geophysics, particularly for environmental issues, for instance for delineating landfills or detecting leachate percolation. Because the reliability of IP data remains an issue at the field scale, this paper deals with the factors controlling data quality and compares different arrays and acquisition parameters for optimal collection of data in the field.

The first part focuses on repeatability experiments carried out in the former Hørløkke landfill (Denmark), in order to infer the degree of which a signal can be reproduced over time. Results show a good repeatability, with on average less than $10 \%$ of difference in raw data. Also, from the results it is inferred that the paramount parameter controlling repeatability is the IP signal level; a value of $2 \mathrm{mV}$ is a sufficient threshold to ensure repeatability within $10 \%$ of data difference, although system dependant.

The second part focuses on survey design and underlines the importance of keeping the geometrical factor low. This points to the choice of a relevant measurement protocol, which depends on the threshold of the geometrical factor, again depending on expected chargeability and resistivity, threshold voltage and injected current. Furthermore, acquisition parameters such as the duration of the pulse injection and data sampling have a significant effect on both the signal-to-noise ratio and resolution. A comprehensive comparison between three protocols, the gradient array, the linear grid and the dipole-dipole array, is shown and the choice of an acquisition sequence is discussed.
\end{abstract}

\section{INTRODUCTION}

The Spectral Induced Polarization (SIP) technique is widely used in environmental geophysics, including detection and mapping of contaminant plumes (Vanhala et al. 1992; Kemna et al. 2004; Sogade et al. 2006), landfill delineation and characterization (Carlson et al. 2001; Johansson et al. 2007; Leroux et al. 2010; Gazoty et al. 2012a) or lithological discrimination (Gazoty et al. 2012b). Field SIP measurements can be carried out either in the frequency domain (Vanhala 1997; Hördt et al. 2007; Williams et al. 2009) or in the time domain (Tombs 1981; Johnson 1984; Slater and Binley 2006; Slater et al. 2010; Doherty et al. 2010; Vaudelet et al. 2011; Fiandaca et al. 2012) with the term 'spectral' referring to measurements at multiple frequencies or at several time channels after a transmitter current shut-off (Hördt et al. 2006). Nowadays commercial geoelectrical equipment often allows the measure of both Direct Current (DC) and Time-Domain Spectral IP (TDSIP or more commonly TDIP) data and the demand for tomographic investigations has driven instrument development towards acquisition systems that switch automati-

*aurelie.gazoty@geo.au.dk cally the electrical connections of the quadrupole sequence by means of relays and multi-channel cables. The use of relays, multi-channel cables and connectors imposes stronger limitations on the maximum voltage applicable by auto-switching geoelectrical systems for current injection, when compared to manual switching systems. Typically the maximum voltage is below $1000 \mathrm{~V}$, to prevent electrical breakdown in relay, cable and connector insulators, e.g., $800 \mathrm{~V}$ for Syscal-Pro equipment (IRIS Instruments), $600 \mathrm{~V}$ for Terrameter LS (ABEM Instrument AB) and $400 \mathrm{~V}$ for SuperSting R8 IP (AGI Advanced Geosciences Inc.), just to name some widely used commercial instruments. For field applications, with ground resistance typically above $1 \mathrm{k} \Omega$, such voltage limitation implies restrictions also on the delivering power for current injection, usually well below $1 \mathrm{~kW}$ (e.g., 250W for Syscal-Pro and Terrameter LS, $200 \mathrm{~W}$ for SuperSting R8 IP). The auto-switching capability of geoelectrical instruments allows to significantly reduce the acquisition time of tomographic $2 \mathrm{D}$ and 3D surveys, especially when combined with multi-channel acquisition. Nevertheless, the need of long quadrupole sequences in tomographic investigations usually limits the stack size of each quadrupolar measurement. Considering the notably smaller signal 
level (in terms of measured voltage) of TDIP data when compared to corresponding DC ones, the low signal-to-noise ratio of TDIP the data often overshadows the potential benefits of the method when using auto-switching instruments and small stack size. A few studies have discussed noise sources in DC and IP data such as the electrode contact resistance (Zonge and Hughes 1985), the composition of electrodes (LaBrecque and Daily 2008) and the electrode polarization effect or the capacitive coupling between cables (Pelton et al. 1978; Dahlin et al. 2002; Radic 2004) and some of them have suggested arrays and designs to minimize these effects (Dahlin et al. 2002; Dahlin and Zhou 2002; Wilkinson 2012). All these noise sources contribute to the noise level of TDIP data, together with the instrumental noise in data sampling and conversion. The present study aims at quantifying the degree of repeatability of TDIP data with auto-switching equipment (i.e., Syscal-Pro, IRIS Instruments) and small stack size. The quantification of data repeatability is not performed in order to suggest field procedures for noise quantification but aims at identifying the factors controlling repeatability in general, in order to give indications for designing TDIP surveys. Indeed, obtaining repeatable data is the minimum demand for having confident results, even if simple repeatability may not be a good measure of data uncertainty in presence of significant systematic errors (LaBrecque et al. 1996; Ramirez et al. 1999; Slater et al. 2006).

In this paper we first discuss data repeatability and we aim at quantifying the parameters controlling it. We do this by analyses of three different experiments designed to cover different aspects of repeatability.

Secondly, we discuss survey design and acquisition parameters affecting the data quality. For this purpose a field comparison of three arrays was carried out to compare the effect of varying quadrupole sequences on noise content, electrode polarization and resolution.

\section{MATERIALS AND METHOD}

\section{Basic principles of TDIP measurements}

In TDIP a voltage decay resulting from an exciting current pulse is measured (see Fig. 1). After the current is turned on, a potential $V_{\mathrm{i}}$, raises immediately across the potential electrodes. After a charge-up effect, the primary voltage, $V_{\mathrm{DC}}[\mathrm{V}]$, is measured for computation of the direct current resistivity just before the current is turned off.

When the current is turned off, the voltage drops to a secondary level, $V_{\mathrm{s}}$ and then decays with time during the relaxation period. This decay curve is characteristic of the medium (in terms of initial magnitude, slope and relaxation time) and represents the target of TDIP.

The signal $V_{\text {IP }}[\mathrm{mV}]$ along the decay is usually integrated over $\mathrm{n}$ time windows or gates for the computation of the chargeability $M$ [mV/V] (Schön 1996; Slater and Lesmes 2002):

$M_{i}=\frac{1}{V_{D C} \cdot\left[t_{i+1}-t_{i}\right]} \int_{t_{i}}^{t_{t+1}} V_{i p} d t$ where $t_{\mathrm{i}}$ and $t_{\mathrm{i}+1}$ are the open and close times [s] for the gate over which the IP signal is integrated.

Hereafter, throughout all the text, the term IP signal refers to the voltage $V_{I P}$, while the term apparent chargeability refers to $M$.

\section{Data sampling}

There are different ways of defining the gate length. With loggating, which is usually done for transient electromagnetic measurements (e.g., Effers $\varnothing$ et al. 1999), the input chargeability is integrated over time intervals whose lengths increase logarithmically with time (Christiansen et al. 2006). This way of integrating the IP signal yields a significant increase of the signal-tonoise ratio by decreasing the standard deviation of the noise with time by a factor of the square root of the gate length (Munkholm and Auken 1996). Indeed, the signal-to-noise ratio is not identically distributed over the time range: the information content is denser in high frequencies, with a high signal-to-noise ratio and poorer in low frequencies (where the decay curve tends to an asymptotic behaviour), with a lower signal-to-noise ratio. The log-gating sampling enables to take these changes into account, as it densely samples the high frequencies and averages the signal over larger gate lengths in the low frequencies.

Figure 2 shows a comparison between log- and linear-gating, for a signal recorded over $4 \mathrm{~s}$. The log-gating technique was used with the gate sampling described in Table 1. The linear sampling is illustrated with two different gate lengths: it covers the $4 \mathrm{~s}$ length with 20 gates of $190 \mathrm{~ms}$ each and 196 gates of $20 \mathrm{~ms}$. The lineargating using $20 \mathrm{~ms}$ gate length returns a good signal quality in the high frequencies (from $0.025 \mathrm{~ms}$ to $1 \mathrm{~s}$ ) but with an increasing deterioration of the signal-to-noise ratio in the low frequencies, beyond $1 \mathrm{~s}$. The linear-gating using $190 \mathrm{~ms}$ gate length keeps a rather good signal-to-noise ratio at late times but loses significant information content in the high frequencies, because it averages different signal levels in the early times. Another way to obtain high signal-to-noise ratio data is to integrate the output signal from

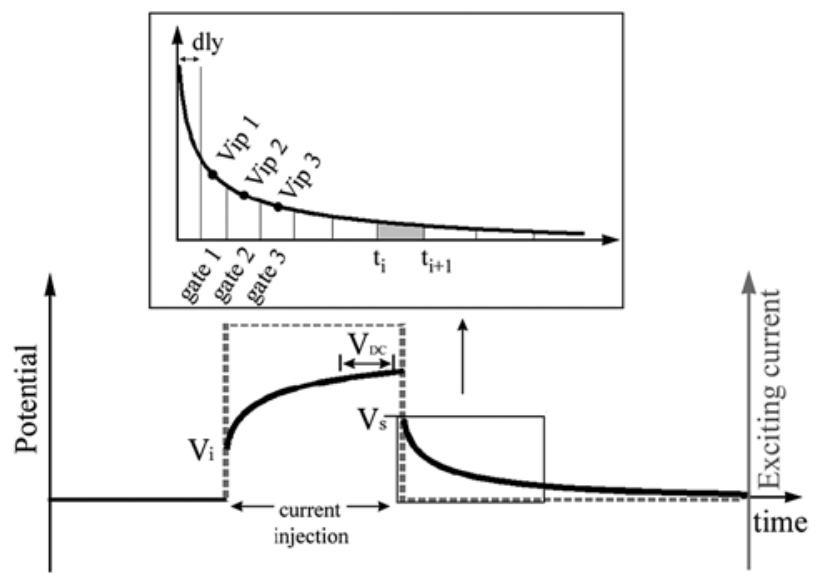

FIGURE 1

Basic principles of TDIP acquisition. The figure shows a sketch of the exciting current and the resulting voltage. 


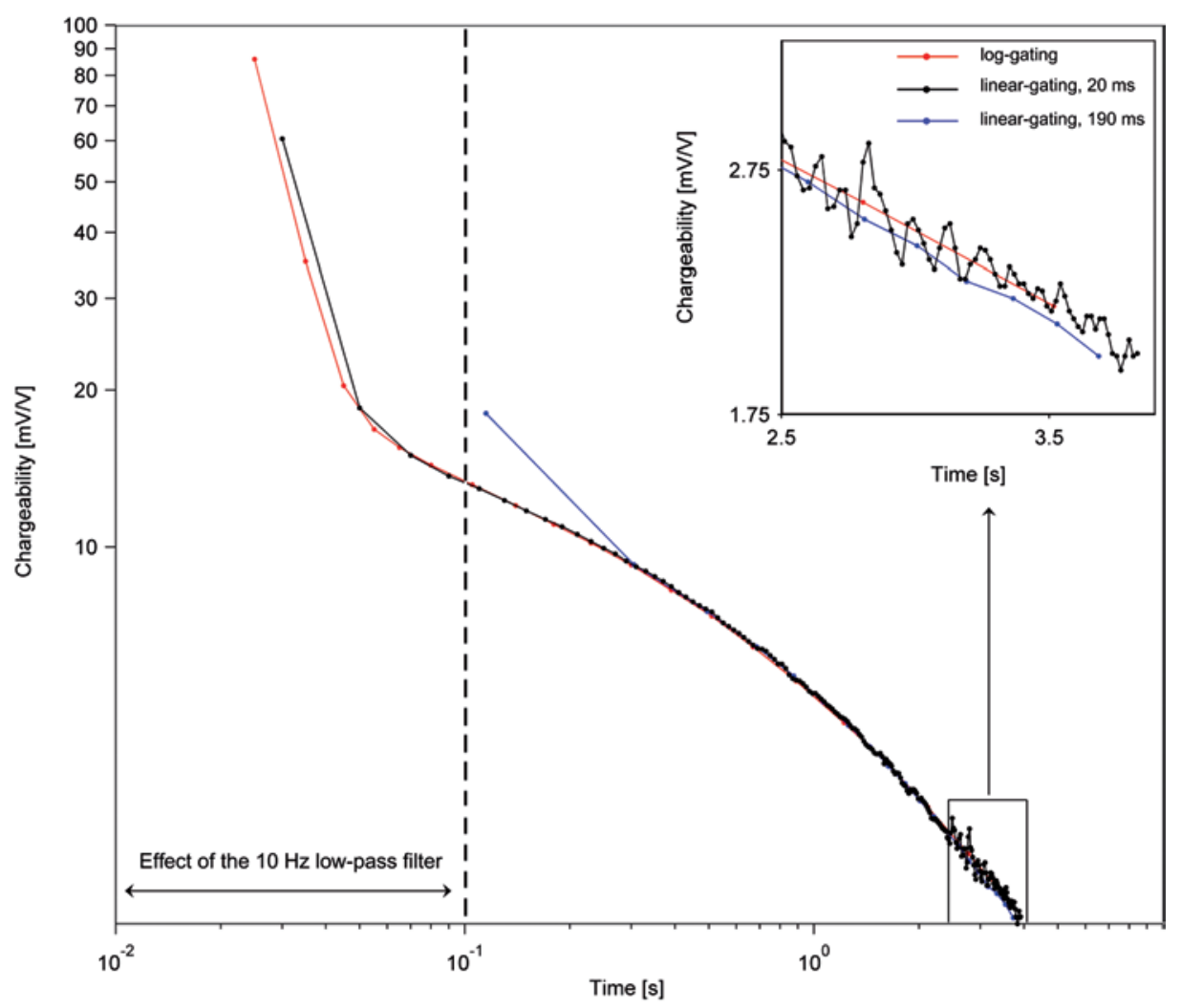

FIGURE 2

Comparison between log- and linear-gating samplings. The signal is recorded with a Syscal-Pro instrument that encompasses a $10 \mathrm{~Hz}$ low-pass filter. high-sampled linear-gating at late times after acquisition, the data quality being then comparable with log sampling. In this case, the limitation comes often from the instrument because it is generally possible to record only a few tens of gates, which is not enough for getting an equivalent signal at late times.

\section{The survey}

The field DC/IP experiments were carried out in the former Hørløkke landfill, in the vicinity of Vojens, Denmark (Fig. 3). The Hørløkke landfill was active from 1968-1978 and the geology of the surrounding area consists of different sand layers with varying clay content and a till-clay layer at $50 \mathrm{~m}$ depth supporting a pollution plume, as described in detail in Gazoty et al. (2012b). Currently, the landfill is neither a bioreactor, nor a site where methane or gas production has been observed and the overall dynamic is very stable. The geophysical survey described in Gazoty et al. (2012b) and performed prior to this study, aimed at delineating the landfill and characterizing the lithology of the surrounding area by means of DC and IP measurements. The results showed a clear signature in IP coming from the landfill, with much smaller apparent chargeability values in the surrounding area.

The signal-to-noise ratio in the raw data was decent throughout the entire site, which was inferred from the visual inspection of each decay curve of the whole data set. The major part of the quadrupole measurements displayed smooth decay curves, with good spatial correlation throughout the acquired profiles and without clear coupling effects. For these reasons the site was chosen as suitable for making both the repeatability experiments and a comparison between protocols. A $410 \mathrm{~m}$ profile exceeding the landfill boundaries (Fig. 3b) was laid out in order to cover both the lowand high-chargeability areas. The measurements were carried out with 83 stainless steel electrodes, $5 \mathrm{~m}$ spaced. The gradient array (Dahlin and Zhou 2006) was used to perform the repeatability experiment, the linear grid array (Fiandaca et al. 2005; Capizzi et al. 2007; Martorana et al. 2009; Capizzi et al. 2010) and the dipoledipole array were used for comparison with the gradient. The settings of the sequences generated with the mentioned arrays are described in Appendices A1, A2 and A3. All the arrays were implemented on a Syscal-Pro (IRIS instruments) set to always apply the maximum output voltage $(800 \mathrm{~V})$ for current injection in order to enhance the signal-to-noise ratio of the data. Three repetitions of the injection cycle were performed for each quadrupole with on- and off-time lengths of $4 \mathrm{~s}$ for the injection and relaxation times. The data were acquired using a log-gating technique ( 8 gates per decade) with 20 gates in total from $25-3520 \mathrm{~ms}$ covering the full IP decay (Table 1). In order to use properly the signal at early times (up to $0.1 \mathrm{~s}$ ), it is important to consider that the Syscal-Pro instrument encompasses a $10 \mathrm{~Hz}$ low-pass filter: the filter effect is modelled in the inversion scheme used in this study (Fiandaca $e t$ al. 2012). In fact, without modelling the filter characteristics, the filter effect at early times could be erroneously interpreted as polarization effects. On the other hand, starting the chargeability sampling after the end of the filter effect, e.g., after $100 \mathrm{ms,}$, would decrease the spectral information contained in the data. 


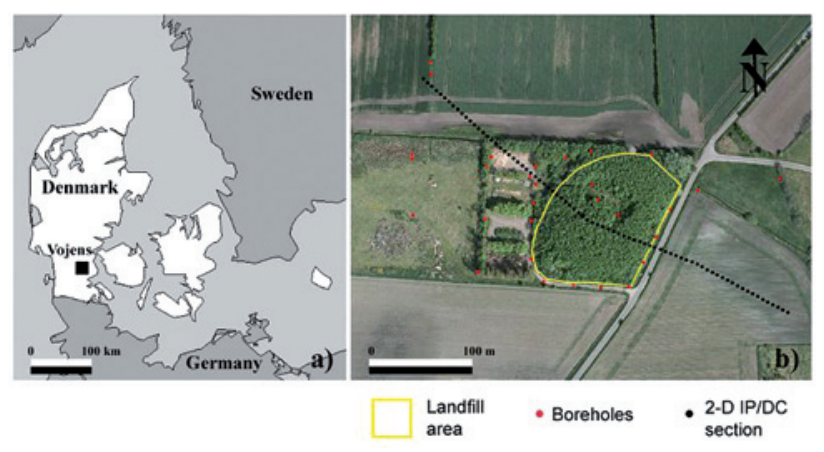

FIGURE 3

The survey area. a) Location of Vojens in the southern part of Denmark. b) IP/DC section (black dots) performed in the former Hørløkke landfill (yellow area). Copyright $($ C Cowi.

\section{REPEATABILITY}

The relatively high noise level of the IP data and the resulting difficulty to assess the data reliability often overshadow the potential benefits of TDIP. The minimum requirement for a reliable method is the possibility to repeat the data in strictly identical conditions: same location, same layout, same settings (with no medium modifications, of course). An equal important issue is to infer the survey repeatability, i.e., with small modifications in the layout or in the medium. Furthermore, from our experience when repeating a survey, the differences in the acquisition settings affect the data more than the changes in the medium. It is then crucial to recognize the critical settings that determine the IP signal.

With these considerations, a repeatability experiment was carried out in the former Hørløkke landfill. The acquisition was repeated with delays of one and two days. One set of experiments consisted in setting the cables and electrodes at the same location, left on the premises with connectors. For the 2 days delay, an additional test was carried out by performing a systematic electrode shift to the side of approximately $20 \mathrm{~cm}$ from their previous location, all in the same way, in order to maintain the same geometry. Considering the landfill size and the local geology (sub-horizontal sandy layers), we expected to map the same lithology with such a small shift. However, the shift was introduced in order to take the variability of the close electrode surroundings into account in the repeatability study. In fact, by shifting the electrodes, we investigate different near-surface effects that might affect the IP signal i.e., different contact, different electro-chemical effects nearby electrodes, different depth at which the electrodes are placed, etc. Thus, shifting the electrodes a few centimetres gives an indication of the general repeatability of a survey. In addition, a comparison with data acquired for a preliminary study thirty days before the repeatability experiment was performed, in order to investigate the repeatability of the survey with small changes to the subsurface. The electrode positioning accuracy for these measurements was about $50 \mathrm{~cm}$.
The next three sections deal with the estimation of data repeatability and try to decipher both the factors controlling repeatability and its connection with standard deviation. Then, it is shown how some acquisition settings may affect significantly the IP signal and the apparent chargeability values. Finally, the effect of the data difference is shown in model space, comparing the inversions of data taken as reference and repeated data.

\section{Estimation of data repeatability}

DC and TDIP data were acquired with the same quadrupole sequence and acquisition settings described in the previous sections. The injected current was $0.4+-0.1 \mathrm{~A}$ for the reference acquisition, with negligible differences of current values in the repetitions performed with a delay up to 2 days, including the experiment carried out with a $20 \mathrm{~cm}$ shift of the electrodes. The stability of the current injection among the different repetitions is due to the standardized instrumental settings (always maximum voltage output, i.e., $800 \mathrm{~V}$ ) and to the homogeneity of the cover soil of the landfill and its surroundings (with contact resistances in average equal to $2.2 \mathrm{k} \Omega$, not varying significantly, also when shifting the electrodes).

In order to quantify the data repeatability, the percentage of difference in apparent chargeability has been computed for each repetition, taking day 1 as a reference:

$\Delta M^{\text {gateX }}=\frac{M_{\text {day } N}^{\text {gate }}-M_{\text {day } 1}^{\text {gate }}}{M_{\text {day } 1}^{\text {gate }}}$

where $M^{\text {gate } X}$ is the chargeability $[\mathrm{mV} / \mathrm{V}]$ for the $\mathrm{X}^{\text {th }}$ gate.

Considering that the chargeability values decrease considerably along the IP decays, we select gate $5(65 \mathrm{~ms})$, gate 10 (230 $\mathrm{ms})$ and gate $15(890 \mathrm{~ms})$ as representatives of the entire time range. Note that some effect from the low-pass filter remains at gate 5 but because of the order of the filter, this effect is negligible. The results are presented in Fig. 4. For all repetitions, the histograms display a narrow distribution centred on zero, with most of the measurements within the interval 0-5\%.

As expected, the early time gate (gate 5) is the most repeatable. The mean differences are $7 \%, 9 \%$ and $10 \%$ for repetitions $1-3$, respectively (mean computed on all three gates at the same time). These results indicate a good repeatability for the IP data, even though the shape of the histograms in Fig. 4 and their long tails involve non-single Gaussian distributions.

The small increase of data difference between the reference acquisition and repetitions 2 and 3 (9\% for 2 days of delay and $10 \%$ for 2 days of delay + electrode shift, respectively), when compared to repetition 1 ( $7 \%$ for 1 day of delay), seems to depend on the different times elapsed between the acquisitions. In fact, the comparison of repetition 3 against repetition 2 presents the same averages and statistical characteristics as the comparison of repetition 1 against the reference acquisition. This suggests that the electrode shift introduces negligible variations in the data, at least when the top soil does not present significant heterogeneity, the contact resistances between two survey repeti- 
tions are comparable and the underlying medium does not suffer modifications.

On the contrary, Fig. 5 shows the comparison between the reference acquisition and repetition 4 , performed thirty days earlier. Repetition 4 displays a wider bell-shape, which is slightly skewed towards negative values and with a mean difference equal to $17 \%$ (for the three gates). In this case, the differences in
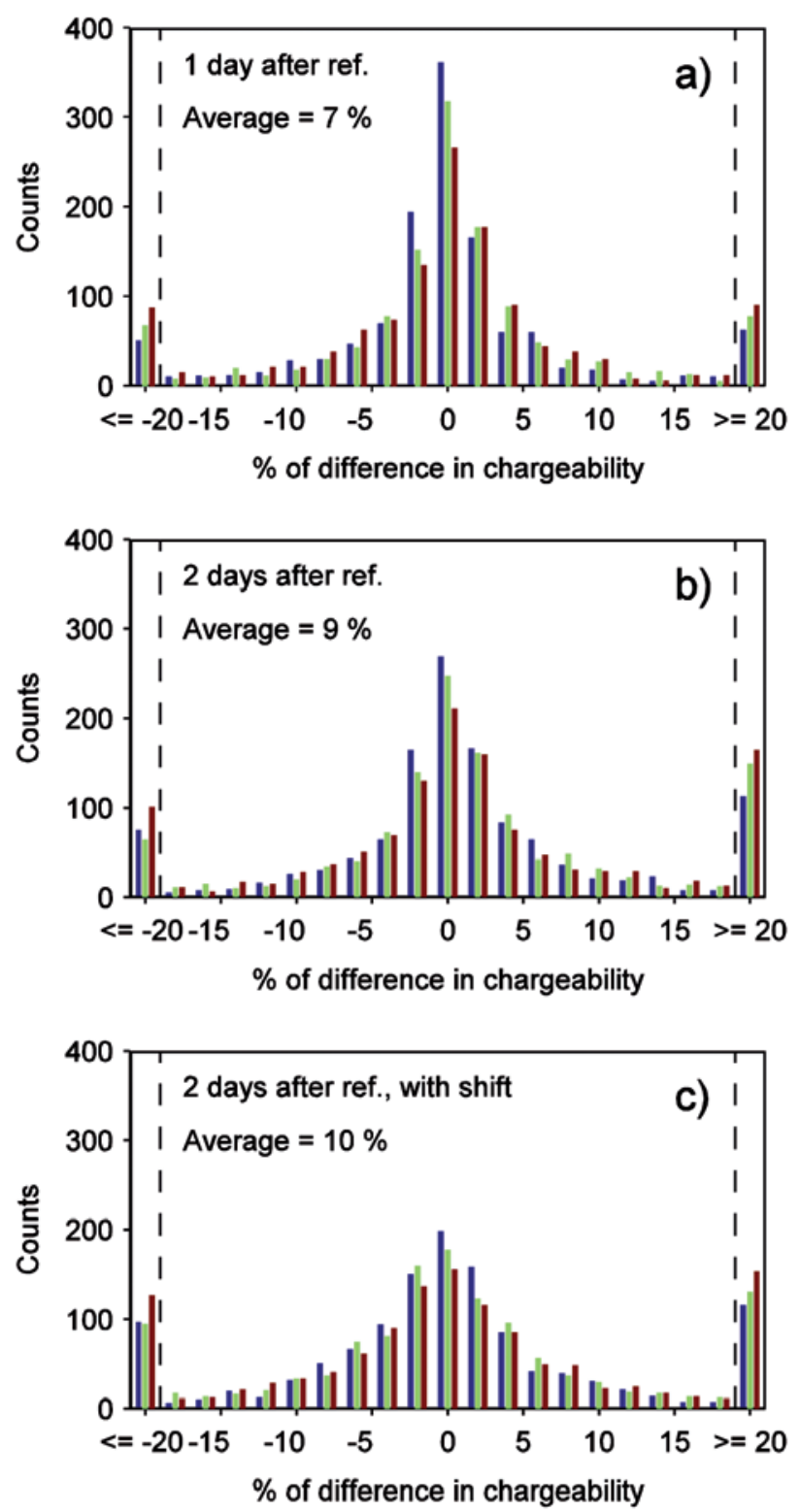

FIGURE 4

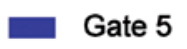

Gate 10

Gate 15

Histogram showing the percentage of difference in chargeability for gates 5, 10 and 15, for each repetition. The measurements were performed with the gradient array. Note that $20 \%$ bins include anything larger than 20\%. a) Repetition 1. b) Repetition 2. c) Repetition 3. chargeability reflect both difficulty in reproducing the data and variations occurred in the underlying medium, probably because of seasonal effects. However, the pseudosection of the IP differences roughly displays the same patterns as the other repetitions, the main differences being located in the shallow part.

\section{Factors controlling data repeatability}

Having quantified the data repeatability, it is important to understand the factors controlling it. Figure 6 shows the distribution of the histogram presented in Fig. 4 on a pseudosection for repetition 1, gate 5. Lateral and vertical focus points are defined to be where $50 \%$ of sensitivity for a homogeneous half-space is met (computed in this way for all other following pseudosections), as stated by Barker (1989) for the vertical focus. Both resistivity and chargeability differences are presented. The repeatability of the resistivity is good, with a difference below $1 \%$ in most of the pseudosection and within $5 \%$ in the shallowest parts. We already saw in Fig. 4 that the differences in apparent chargeability for repetition 1 are $7 \%$, on average. When looking at the distribution of these differences on a pseudosection (Fig. 6b), it is clear that the differences are not randomly laid out. Indeed, the biggest difference mainly occurs in the left part of the section, below $10 \mathrm{~m}$ depth. This was observed systematically for all repetitions (not shown here).

The Syscal-Pro instrument provides in addition to the DC and chargeability data a measurement of the self-potential, which is the voltage measured without any external excitation source (affected also by soil-electrode interactions when using polarizable electrodes, as in our case). We tried to infer whether the observed difference in chargeability could be due, to some extent, to the measured self-potential. No correlation was found between this pattern and the self-potential signal. Furthermore, no correlation could be established either between the data repeatability and the time of re-use of an electrode for potential measurement after

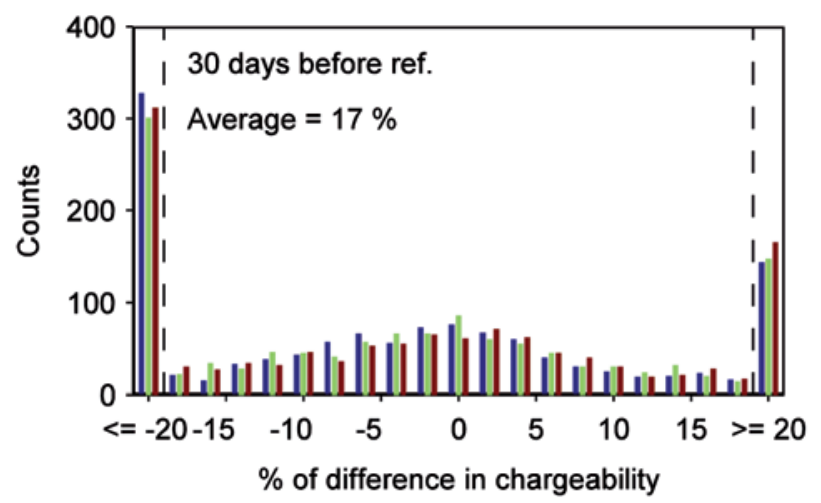

Gate 5

Gate 10

Gate 15

FIGURE 5

Histogram showing the percentage of difference in chargeability for gates 5,10 and 15 , for a measurement performed 30 days before the reference. Note that $20 \%$ bins include anything larger than $20 \%$. 

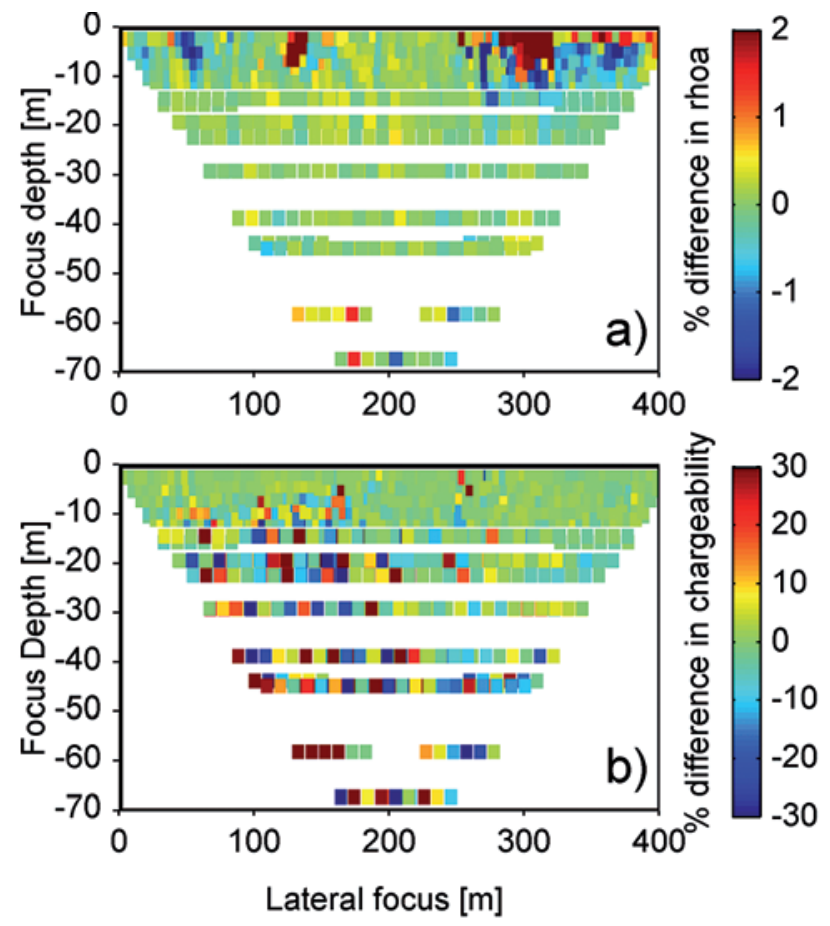

FIGURE 6

Pseudosection showing the percentage of difference in the collected data, for repetition 1. a) In apparent resistivity. b) In chargeability for gate 5 . Note that the colour scales are different, the DC scale ranges from -2 to 2 , while the IP chargeability ranges from -30 to 30 .

having injected current (i.e., the electrode polarization effect).

Figure 7 shows the distribution of the IP signal $V_{\text {IP }}$ (see Fig. 1), in comparison with the distribution of the chargeability differences. These two plots clearly display similar features and show that the data repeatability and IP signal level are closely linked to each other. Indeed, wherever the signal level $V_{\mathrm{IP}}$ is high the data repeatability is good (e.g., on the right side of the pseudosection), whereas the data are less repeatable where the signal level is low. Figure 7(c) shows the percentage of difference in chargeability versus $V_{\mathrm{IP}}$, for repetition 1 , gate 5 . The figure indicates that a high IP signal level supports high data repeatability. There is a clear correlation between the two parameters, with a Pearson's correlation coefficient of 0.7. This trend is also observed in repetitions 2 and 3 but not shown here. Figure 7(c) can be used to establish signal thresholds that ensure average repeatability. For instance, the threshold in $V_{\mathrm{IP}}$ read in Fig. 7(c), capable of ensuring less than $10 \%$ of difference in chargeability, is $2 \mathrm{mV}$ (10\% being the range of difference observed in Fig. 4). $\mathrm{V}_{\text {IP }}$ signals below $2 \mathrm{mV}$ might repeat equally as good as the higher voltages but on average they do not. This threshold is specific to the Syscal-Pro instrument used for this study and depends on the stack size in the measurement procedure (three cycles of injection for each measure) as well as the selected gate but it is not site-dependent, as it was consistent for repetitions 2 and 3 and for another field site in Denmark.
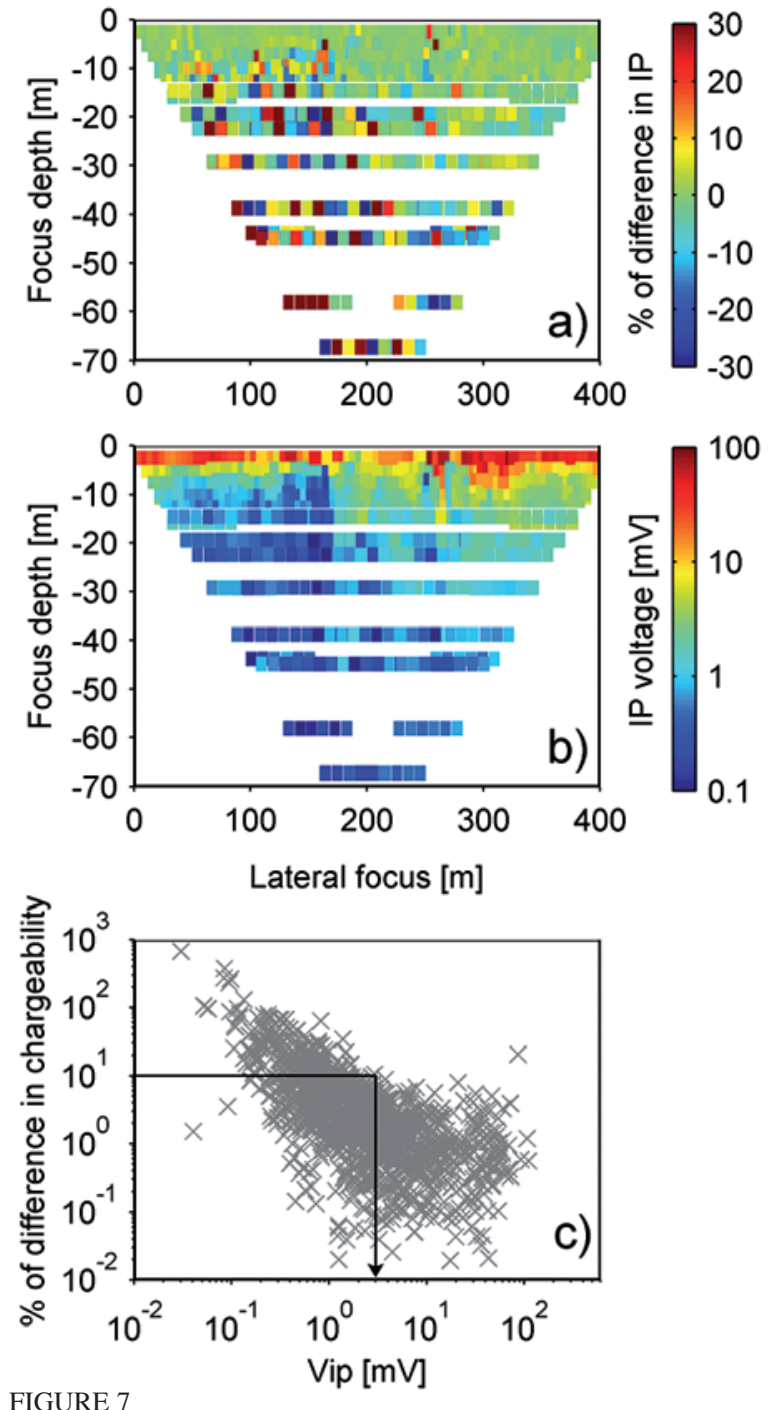

FIGURE 7

Parameters controlling the data repeatability. a) Pseudosection showing the percentage of difference in IP for repetition 1 (gate 5). b) Pseudosection of the $V_{\text {IP }}$ level (gate 5). c) Relation between $V_{\text {IP }}$ and the percentage of difference. The arrow indicates the threshold $V_{\mathrm{IP}}$ defined on the basis of $10 \%$ of difference in chargeability.

\section{Standard deviation as a measure of data repeatability?}

The standard deviation computed on the different cycles of current injection used in the stacking procedure is typically estimated by geoelectrical instruments in the field. Usually it is also the paramount information provided by the instruments to assess the data quality. We considered so far the capability of the data to be reproduced from a complete data set to another, over the same model. But is the repeatability over different stacks of the same measurement comparable to the repeatability over different data sets (at least for strictly identical set-ups)?

The standard deviation provided by the Syscal-Pro instrument is computed on the chargeability integrated over all 20 gates 
(integral chargeability) and therefore represents a mean value for the entire decay. In Fig. 8 we compare the repeatability from repetition 1 and the relative error from the instrument. The relative error, IP_std, plotted on Fig. 8(b) is computed as follows:

$I P_{s t d}=\frac{\sigma_{M}}{M} * 100$

where $\sigma_{\mathrm{M}}$ is the standard deviation provided by the instrument and $M$ the integral chargeability over the 20 gates.

Figure 8(a,b) does not reveal a strong relationship between the repeatability and the relative error, though the overall trends agree in rough terms. Figure 8(c) shows the difference from the repeatability versus relative error and indicates a rough correlation between the two parameters but with large variations. The calculated correlation coefficient is 0.4 , considerably smaller than the one found for repeatability as a function of $V_{\mathrm{IP}}$. In particular, for a very low relative error close to zero (the linear segment represents all low values from the instrument given as zero on the output), the percentage of difference still spans three orders of magnitude, ranging from good to really poor repeatability. We observe the same behaviour for larger relative errors, showing that the standard deviation provided by commercial instruments is not always helpful, or at least sufficient. Part of this can be explained by the way the stacks are computed by the instrument, as shown by Fiandaca et al. (2012). Basically, the remaining IP-potential at the end of the off-time will shift the potential for the next pulse, thus shifting the measured values, which in turn bias the measure of standard deviation up to several per cent (Fiandaca et al. 2012). In this respect the reciprocal measurements provide a more reliable estimation of noise content (LaBrecque et al. 1996; Ramirez et al. 1999; Slater et al. 2006).

\section{Critical acquisition settings that determine survey repeat- ability: current waveform}

The repeatability of an IP survey is not only determined by the accuracy of the electrode positioning and by the modification occurred in the medium during two investigations but also by the settings used for the data acquisition. In particular, the entire current waveform, including the duration of the current pulse and stack size, plays a crucial role in determining the strength and shape of the chargeability decays.

Tombs (1981) and Fiandaca et al. (2012) showed that the IP response, $V_{\text {PULSE }}$, due to a single current pulse of finite duration $T_{\text {ON }}$, is a superimposition of two step responses, $V_{\text {STEP }}$, one positive and one negative shifted in time:

$V_{P U L S E}(t)=V_{\text {STEP }}(t)-V_{\text {STEP }}\left(t+T_{O N}\right)$

where $t$ is the time [s] and $T_{\mathrm{ON}}$ is the pulse length [s].

When the pulse duration $T_{\mathrm{ON}}$ is big compared to the time characteristic of the IP phenomenon, $V_{\text {STEP }}\left(t+T_{\text {ON }}\right)$ becomes negligible compared to $V_{\text {STEP }}(t)$ and the pulse response is approximately equal to the step response. Otherwise, the effect of the pulse length becomes severe on the IP data and significant on the DC ones. Figure 9 shows this effect on the synthetic model shown in Fig. 16, for a representative quadrupole ( $|\mathrm{AM}|=37.5 \mathrm{~m}$ and $\mid \mathrm{MNI}=2.5 \mathrm{~m})$. Other characteristics of the current waveform, e.g., the stack size, also play a role in determining the DC and IP data but to a smaller extent (e.g., Fiandaca et al. 2012).

In the following a field example of the effect of the duration of current pulse on DC and IP data is also shown. The experiment was conducted on an identical medium using exactly the same acquisition set-up, stack size and quadrupole sequence but with different injection pulses of $1 \mathrm{~s}$ and $4 \mathrm{~s}$. The first 14 gates of Table 1 were used identically for the first second of measurement
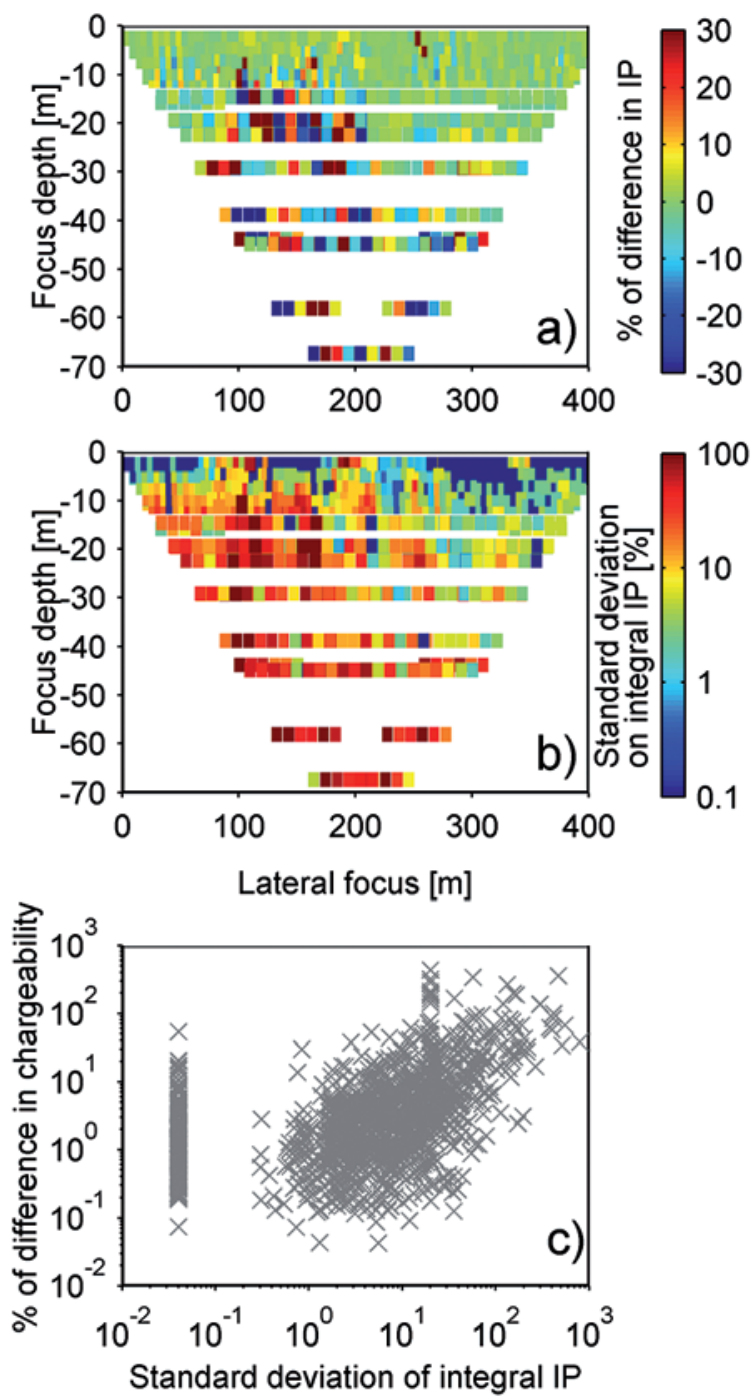

\section{FIGURE 8}

Assessment of data repeatability. a) Percentage of difference in chargeability. b) Standard deviation. c) Repeatability versus relative error. Note that the linear segment present for a standard deviation between $10^{-2}$ and $10^{-1}$ represents all low values from the instrument given as zero on the output. 
in both experiments for measuring the decay curves, this is why a comparison between IP data sets is made possible (Fig. 9c). Then, the differences, $\Delta$, in the raw data were computed as percentages for both resistivity and chargeability for a selected gate, taking the signal recorded with a $4 \mathrm{~s} T_{\mathrm{ON}}$ as a reference:

$\Delta_{\rho}=\frac{\rho_{1 s}-\rho_{4 s}}{\rho_{4 s}} * 100$

and

$\Delta_{M}^{\text {gate }(X)}=\frac{M_{1 s}^{\text {gate }(X)}-M_{4 s}^{\text {gate }(X)}}{M_{4 s}^{\text {gate }}(X)} * 100$

where $\rho$ is resistivity $[\Omega \mathrm{m}]$ and $M$ is chargeability for a selected gate $X[\mathrm{mV} / \mathrm{V}]$.

The results in Fig. 10(a) show a minor difference in raw resistivity data, within $4 \%$. This difference occurs in the middle of the pseudosection, at the landfill location, where the signal is high. As also shown in the synthetic example Fig. 9(b), the pulse length of $1 \mathrm{~s}$ was not enough to allow the ground to charge-up completely and the DC voltage at $1 \mathrm{~s}$ was lower than the DC voltage measured at $4 \mathrm{~s}$. This is an IP effect measurable in the DC data. The differences in chargeability presented in Fig. 10(b) are at a different scale, ranging from $-35 \%$ to $-50 \%$ for gates 3-9 (Table 1) and more than $-100 \%$ at later times (not shown in the figure). The negative sign of the differences for chargeability were expected.

Consequently, using the same current waveform is essential when performing repeatability studies involving chargeability as well as resistivity and considering the effect of the current waveform in the forward response is necessary for a quantitative interpretation of the IP data.

\section{Repeatability in model space}

It has been shown in Fig. 4, that in our case, 7-10\% of difference in the raw data was measured when repeating the IP acquisition. Considering these values, what can be expected after inversion in terms of comparison between models? Figure 11 shows the inverted models for data from the reference day and 2 days lapse with shift, which is, repeatability wise, one of the most pessimistic cases. The data sets were independently processed by removing outliers and inverted independently following Fiandaca et al.
(2012). This new inversion scheme inverts the TDIP data directly for the Cole-Cole parameters. The time-domain forward response is computed via a Hankel transform of the frequency domain response for a layered medium. The low-pass filter modelling is included so that the calculated forward response is subject to the same filtering as the measured response. The impedances of the layers are computed from the Cole-Cole model (Pelton et al. 1978). Then, the inversion is carried out with 1D-LCI implementation (Auken et al. 2005), to retrieve the four Cole-Cole parameters (resistivity, $c, \tau$ and $M_{0}$ ), for each layer. The standard deviation used in the inversion process was set equal to $3 \%$ for DC resistivity data and $10 \%$ for IP data, these two values based from the hereby repeatability survey.

From Fig. 11, three main features can be identified: the waste layer can be clearly seen in the $M_{0}$ section, displaying a shallow highly chargeable unit of more than $100 \mathrm{mV} / \mathrm{V}$. The agreement between this layer and the borehole is good in terms of depth and thickness, for both the reference day and 2 days lapse. On the western part, the clay layer agrees with a conductive body of 20-30 $\Omega \mathrm{m}$ and again, both sections are in good agreement in this respect. The peat lens present at $100 \mathrm{~m}$ from the west boundary is also present in both sections, with a higher chargeable unit of $100 \mathrm{mV} / \mathrm{V}$. Overall, the models are very similar and the main features are mapped accurately. Minor differences are present at depth due to a lack of resolution in $M_{0}$, which explains also the differences of $c$ and $\tau$ below $15 \mathrm{~m}$ depth.

These results indicate that the range of differences observed in the raw data $(10 \%)$ does not affect the models in terms of interpretation. This is most likely because the errors are not correlated and they compensate each other to some extent.

\section{SURVEY DESIGN}

The efficiency of performing a survey, in terms of time consumption, gained information and general costs, highly depends on the choice of the instrumentation set-up. This includes the array, commonly implemented on a multi-channel acquisition system, the acquisition parameters such as the pulse length $\left(T_{\mathrm{ON}}, T_{\mathrm{OFF}}\right)$, the stack size, the injected current, the choice of performing reciprocal measurements or not, etc. The survey design is usually a balance between acceptable data quality, gained information and a reasonable acquisition time. Recently, Wilkinson et al.

\section{FIGURE 9}

Sketch showing different pulse lengths of $1 \mathrm{~s}$ and $4 \mathrm{~s}$ for the measurement of IP/DC data. The waveform is computed for the three-layer model shown is Fig. 16. a) $T_{\mathrm{ON}}$ and $T_{\text {OFF }}$ b) Zoom on the On Time, where the DC data are computed. c) Zoom on the Off Time, where the time decays are measured.
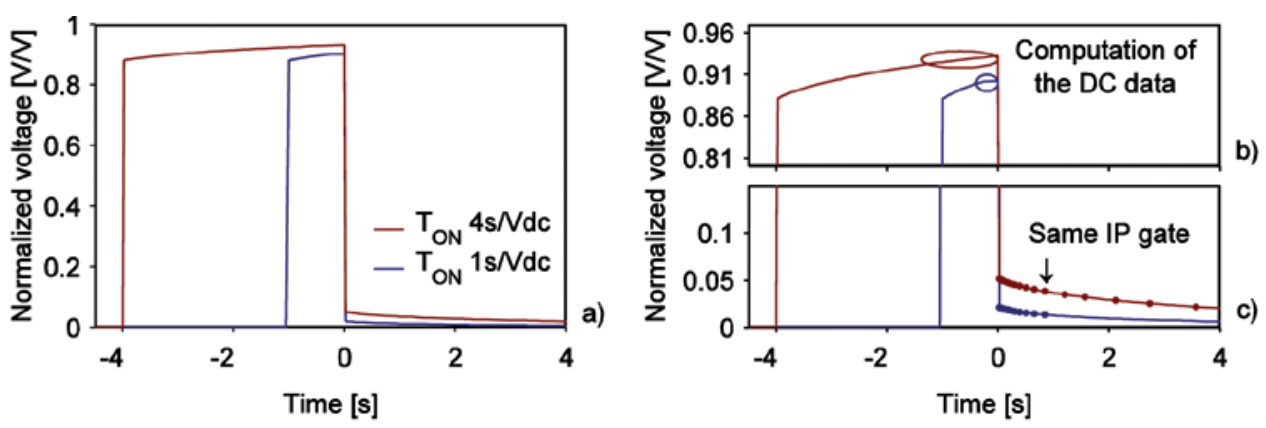

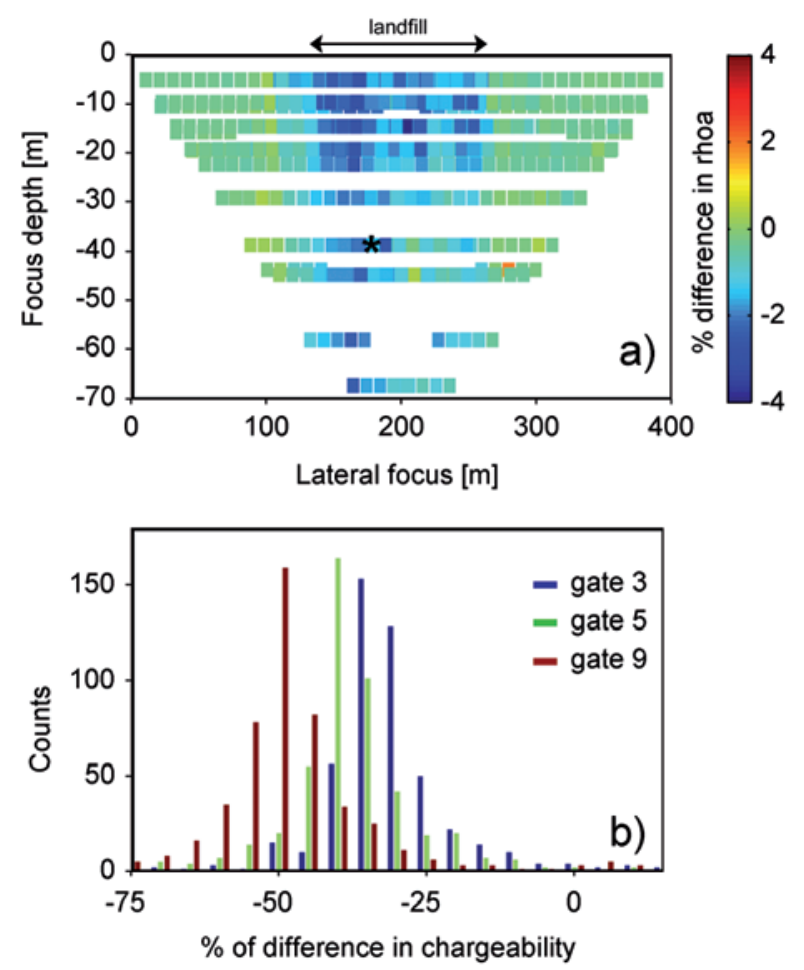

FIGURE 10

Influence of the pulse length on the data repeatability. a) Difference in resistivity plotted on a pseudosection. The star refers to the location of the chargeability data presented in Fig. 15. b) Difference in percentage on chargeability for three different gates.

(2012) provided a review for optimizing resistivity surveys, including protocol design, minimization of electrode polarization effect and estimates of data noise. The present study deals with different considerations and is more focused on acquisition parameters, for both IP and DC data. In the next section the effect of the threshold on the signal level, in terms of measured potential in the decay curve, is investigated. In particular its effect on the survey design and the choice of arrays is detailed and an illustrative comparison among three arrays, well-suited for IP surveys is presented. In the second and third sections the effect of the length of the injection pulse $T_{\mathrm{ON}}$ is discussed, both for signal-to-noise ratio and information content.

\section{Current injection and geometrical factor}

In the previous section it was shown that the paramount parameter controlling data repeatability is the signal level $V_{\text {IP. }}$. In order to illustrate the factors influencing the $V_{\text {IP }}$ level we consider the formulas below for resistivity and initial chargeability for the step response in a homogeneous half-space:

$\rho=k \frac{V_{D C}}{I}$

and
$M_{0}=\frac{V_{I P\left(t=t_{0}\right)}}{V_{D C}}$

where $\rho$ is resistivity $[\Omega \mathrm{m}], M_{0}$ is chargeability $[\mathrm{mV} / \mathrm{V}]$ measured at $t_{0}, t_{0}$ is the time at which the current is turned off, $I$ is the injected current [A], $V_{\mathrm{IP}}$ the IP signal $[\mathrm{mV}]$ and $V_{\mathrm{DC}}$ the potential used for calculating the DC resistivity [V]. The signal $V_{\mathrm{IP}}$ can be expressed combining the two expressions as follows:

$V_{I P\left(t=t_{0}\right)}=\frac{\rho * M_{0} * I}{k}$

Therefore, in order to increase the signal level it is necessary to increase the injected current and/or to decrease the geometrical factor, the soil parameters being independent on the survey design. At the scale of the survey presented in this study, our experience suggests the use of the maximum power selectable with auto-switching instruments for the current injection (typically $250 \mathrm{~W}$ ), even if it implies some complications in power supply management in the field.

The values of the geometrical factor $k$ depend on the array and on the profile layout (electrode spacing and length of the profile): imposing limits on the geometrical factor means imposing limits on the choice and design of the quadrupole sequence. Indeed, for some arrays like the dipole-dipole where the geometrical factor reaches very high values with the dipole offset, the data quality decreases dramatically with depth. On the other hand, the threshold controls the maximum depth of investigation of a survey; so it is necessary to increase the geometrical factor for increasing the depth of investigation. It is therefore crucial to define a reliable range for the geometrical factor when designing the array. From expression (8) it is possible to express the geometrical factor threshold as a function of a required $V_{\text {IP }}$ level, the soil parameters and the injected current:

$k=\frac{\rho * M_{0} * I}{V_{I P\left(t=t_{0}\right)}}$

In the survey design it is then important to choose the array and build the acquisition sequence considering:

1) The expected average resistivity and chargeability of the soil;

2) The signal level $\left(V_{\text {IP }}\right)$ necessary to obtain a good signalto-noise ratio;

3) The amount of current that is possible to inject into the ground.

All these three factors control the choice of a threshold for defining a proper geometrical factor when preparing a sequence of measurements while the last two depend on the actual instrument and the electrode contact.

For instance, if we assume a minimum $V_{\text {IP }}$ level of $2 \mathrm{mV}$ as previously discussed, $0.4 \mathrm{~A}$ for the current (i.e., the average current injected in this study), $180 \Omega \mathrm{m}$ for the resistivity (the average apparent resistivity of the profile) and $100 \mathrm{mV} / \mathrm{V}$ for the 

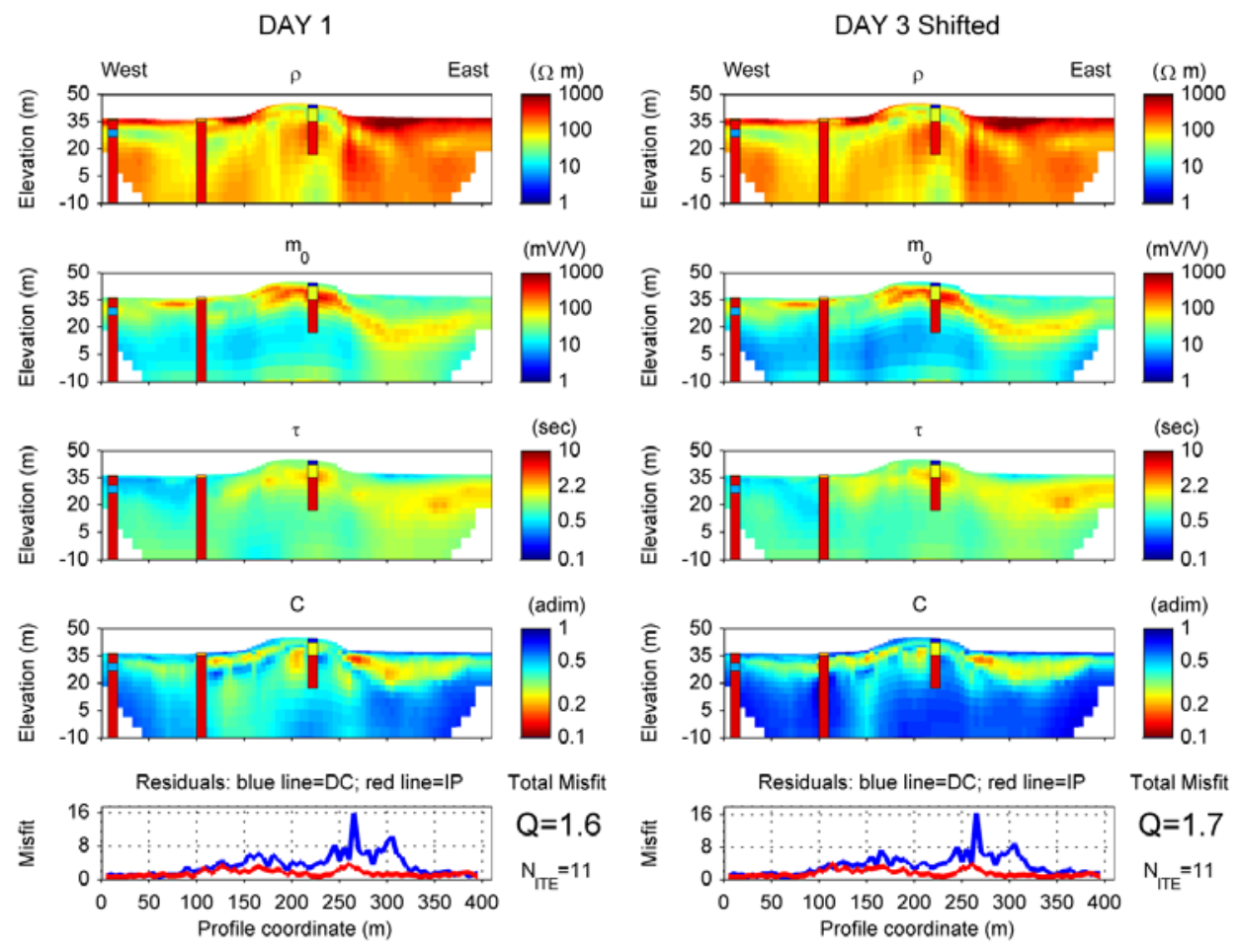

FIGURE 11

Comparison between models for a two day lapse, for the four ColeCole parameters (resistivity, $M_{0}, c$ and $\tau$ ). Boreholes are superimposed to the $2 \mathrm{D}$ sections. The landfill boundaries stand between 150-250 m, where there is topography.

Borehole colorscale:

$$
\text { cover layer waste } \quad \text { sand } \quad \text { clay }
$$

chargeability (the lower value of chargeability for the peat and landfill in the inverted profiles), a maximum value of $3600 \mathrm{~m}$ for the geometrical factor is found.

\section{Arrays and resolution}

In this study, three different arrays were chosen to acquire the profile shown in Fig. 3: the gradient array, the dipole-dipole and the linear grid. These arrays were selected for comparison because all of them are suited for multi-channel acquisition and reduce the influence of the electrode polarization effect (see Appendix A4). Of course, these three arrays differ also in terms of resolution but a systematic comparison on their resolution is beyond the scope of this paper and has been partially answered by Dahlin and Zhou (2004), Fiandaca et al. (2005) and Martorana et al. (2009). The characteristics of the different arrays discussed here are outlined in details in Appendices A1, A2, A3 and Fig. 17. Figure 12 displays the distribution of the geometric factor $k$ on a pseudosection for the three arrays. Figure 13 shows the number of measurements involved when increasing $k$, with the value of $3600 \mathrm{~m}$ highlighted.

The gradient array with 83 electrodes and $5 \mathrm{~m}$ between takeouts has a maximum value for the geometrical factor below $3600 \mathrm{~m}$ by construction. The quadrupole sequence of the linear grid array was built imposing the threshold of $3600 \mathrm{~m}$ for the geometrical factor but compared to the gradient sequence many measurements have $k$ values close to the threshold. Consequently, the choice of the threshold is more critical for the linear grid array when compared to the gradient array. With the dipoledipole array, we rapidly reach the threshold with depth (just below 20 m, Fig. 12b), with only 560 measurements out of 1154 (49\%) below $3600 \mathrm{~m}$ (Fig. 13). Thus, the choice of a relevant protocol highly depends on the threshold of $k$ and it really matters to define a consistent value considering the three parameters above-mentioned (expected chargeability and resistivity, required $V_{\text {IP }}$ level and injected current).

Figure 14 shows the inverted model $M_{0}$, for the three arrays with the corresponding uncertainty analysis. The uncertainty analysis indicates how the parameters are determined and computed by taking the square root of the diagonal elements of the covariance matrix of the last iteration of the linearized inverse problem, following Tarantola and Valette (1982). Lower values indicate better parameter resolution. Due to the large number of constraints on the model we will, in this case, only use the numbers relatively. The three arrays roughly display the main features described above, i.e., the landfill boundaries in the mid-part of the section, the clay layer and the peat lens, in agreement with borehole information. The main differences are located at depth and on the eastern part. Because of the lack of geological information in the east, it is difficult to infer which array provides more realistic mapping. However, the uncertainty analysis indicates that the linear grid provides the best resolution over all the section, followed by the gradient array and the dipole-dipole. As 
shown in details in Appendix A4, differences among arrays exist also in terms of electrode polarization. The linear grid array shows better efficiency in this respect, followed by the dipoledipole and the gradient arrays.

\section{Effect of $T_{\text {oN }}$ on the signal-to-noise ratio}

As previously mentioned, the choice of different pulse lengths affects the data, mostly the chargeability. For a larger $T_{\mathrm{ON}}$, it was shown that the chargeability is expected to be different, with higher values. This result has a direct influence on the data quality, as a higher signal gives a higher signal-to-noise ratio.

Figure 15 shows an example of raw data in chargeability for different pulse lengths of $1 \mathrm{~s}$ and $4 \mathrm{~s}$, the other acquisition parameters being exactly the same (first 14 time gates of Table 1). The decay curve from the $4 \mathrm{~s}$ pulse has higher magnitude and its quality is visibly better than the one using a $1 \mathrm{~s}$ pulse. This happens when the polarization phenomenon has a time characteristic longer than the pulse length and the charge-up effect is not completed during the current injection. Thus, increasing $T_{\mathrm{ON}}$ gives more chances to fulfil the desired threshold requirement for the IP signal level, when the time characteristic of the IP phenomenon is comparable to the duration of the current pulse.
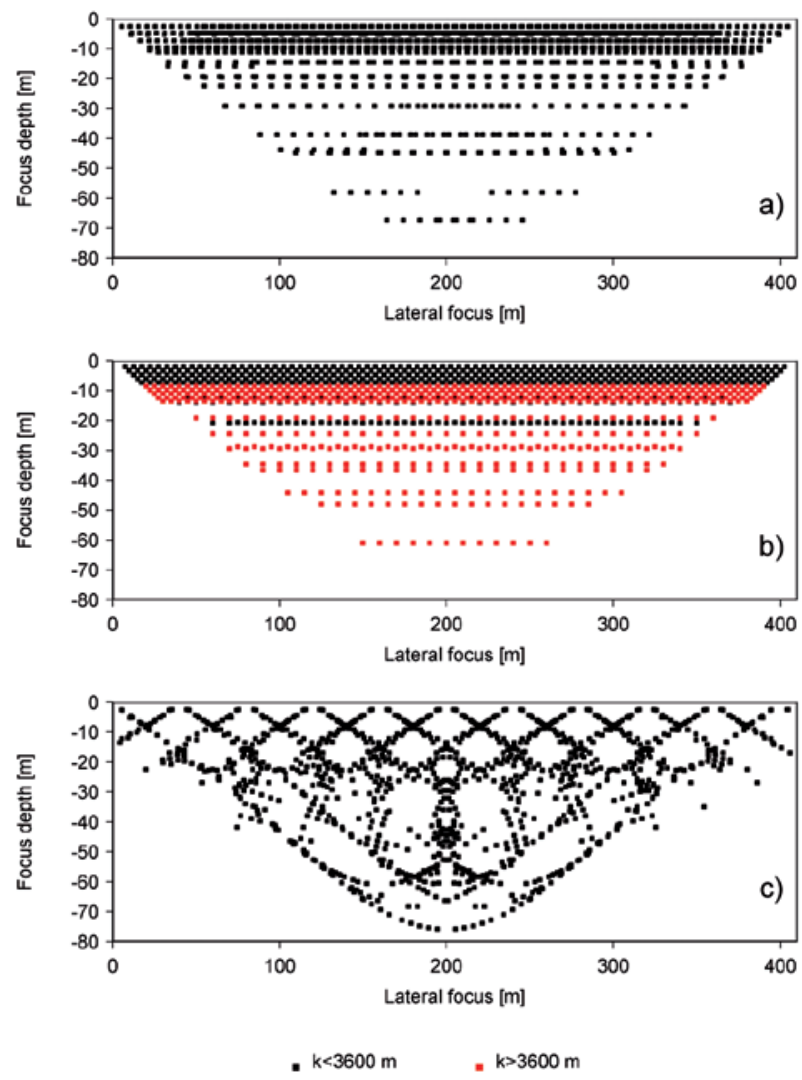

FIGURE 12

Geometrical factors displayed on a pseudosection for three array configurations. a) Gradient array. b) Dipole-dipole. c) Linear grid.
Effect of $T_{\mathrm{ON}} / T_{\mathrm{OFF}}$ on the information content

In order to quantify the effect of $T_{\mathrm{ON}} / T_{\mathrm{OFF}}$ for resolving the inversion parameters (here the four Cole-Cole parameters), a Schlumberger sounding was simulated on a simple synthetic model with three layers. In Fig. 16 the three layer model is shown, with a highly chargeable unit in the middle (red curve). The forward and inverse computations are based on Fiandaca $e t$ al. (2012). Two data sets were computed using a $T_{\mathrm{ON}}$ of $4 \mathrm{~s}$ and $1 \mathrm{~s}$ and a stacking of three current injection cycles. $10 \%$ and $3 \%$ of Gaussian noise were added to the chargeability and resistivity data, respectively. The inversion results are presented in Fig. 16(a,b) (continuous line) and the difference with respect to the true model is presented in Fig. 16(c).

For a longer pulse, the inverted model is closer to the synthetic model, with higher accuracy, as the uncertainty bandwidth (dashed lines) is narrower. For a $4 \mathrm{~s}$ pulse, Fig. 16(c) indicates a difference with respect to the true model in a range of $5 \%$ for parameters $\rho, M_{0}$ and $c$, whereas it reaches $25-30 \%$ of difference with a $1 \mathrm{~s}$ pulse. Thus, the choice of a different $T_{\mathrm{ON}}$ has a significant influence on data repeatability, data quality and the resolution of inversion parameters (furthermore, the higher noise content in chargeability, for data acquired with a smaller acquisition time, was not taken into account in this simulation).

\section{DISCUSSION AND CONCLUSIONS}

The reliability of the TDIP method is based on its capability to be reproduced in time for mapping a geological background unchanged at the survey scale. The limitations on maximum injectable current and stack size have strong implications on the signal-to-noise ratio of TDIP data and require a deep understanding of the data reproducibility. This study quantifies the TDIP data repeatability with a Syscal-Pro instrument in the range of $7-10 \%$, in the framework of experiments conducted on a former landfill by repeating data acquisition over time. The repeatability experiment did not aim at establishing a procedure for error estimation in the field but was used to identify the paramount parameter controlling the data repeatability, i.e., the IP signal level, in

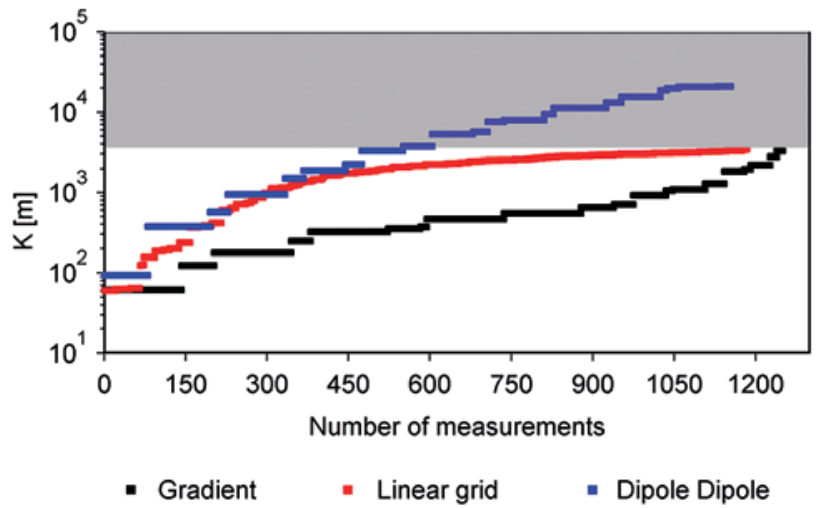

FIGURE 13

Geometrical factor $k$ versus number of measurements for three different arrays. The grey area in transparency fits a geometrical factor above $3600 \mathrm{~m}$. 
FIGURE 14

Comparison between models $M_{0}$ for three different arrays. The landfill boundaries stand between 150-250 m, where there is topography.
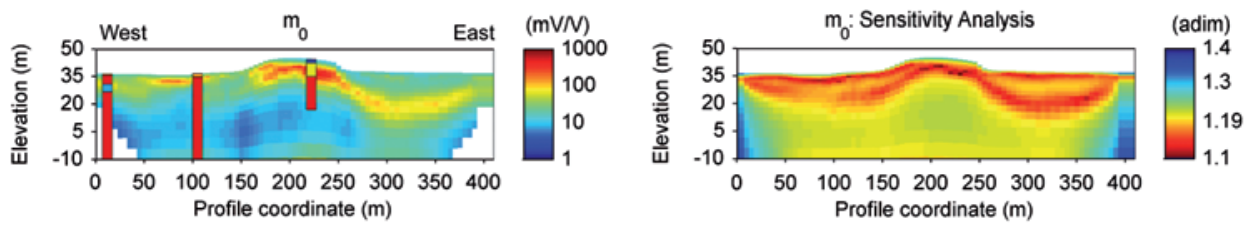

Linear Grid
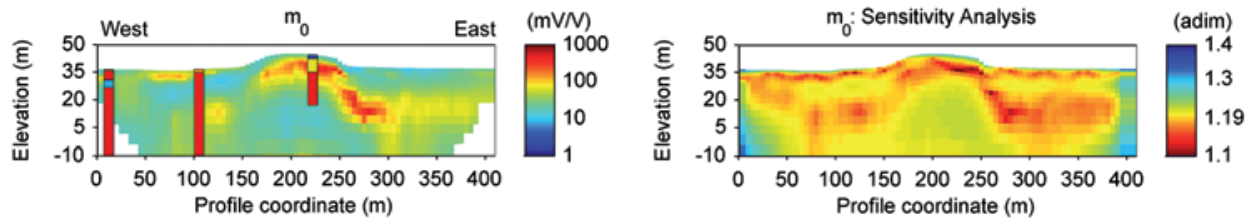

Dipole Dipole
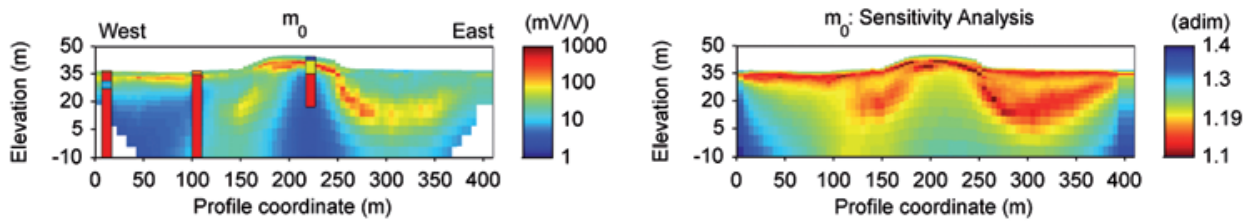

Borehole colorscale:

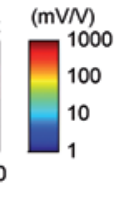

cover layer

waste

sand peat

clay

terms of measured potential in the decay curve. A value of $2 \mathrm{mV}$ could be inferred as a sufficient threshold to ensure $10 \%$ of difference in repeated IP data. Though a significant number of data with an IP signal below this threshold also displays a repeatability lower than $10 \%$, on average they are less repeatable and reliable. Other mechanisms likely contribute to the data quality but they have not been identified in this study. Further studies are necessary to completely address this issue, because the value of the $V_{\mathrm{IP}}$ threshold, as stated now, involves strong limitations in the array design. Furthermore, the magnitude of the threshold also depends on the instrument and on the stack size used in this study. Despite this, the IP signal level remains the key parameter that controls repeatability.

The degree of data reproducibility was also studied in terms of effects on the inversion results. The comparison between inversion models corresponding to different acquisitions shows how data uncertainty propagates into the model space. With $10 \%$ of difference in the raw data the main geological features can be recovered and similar models can be obtained after inversion, the main differences being due to a lack of resolution at depth. Data interpretation is not significantly affected by this level of noise.

Secondly, the paper deals with aspects of the survey design. Having recognized the signal level as the key parameter ensuring data repeatability, it is then crucial to design the survey accordingly. This directly involves the geometrical factor, the level of the current injection and the length of the current pulse. Usually in IP survey design, the resolution and the electrode polarization are

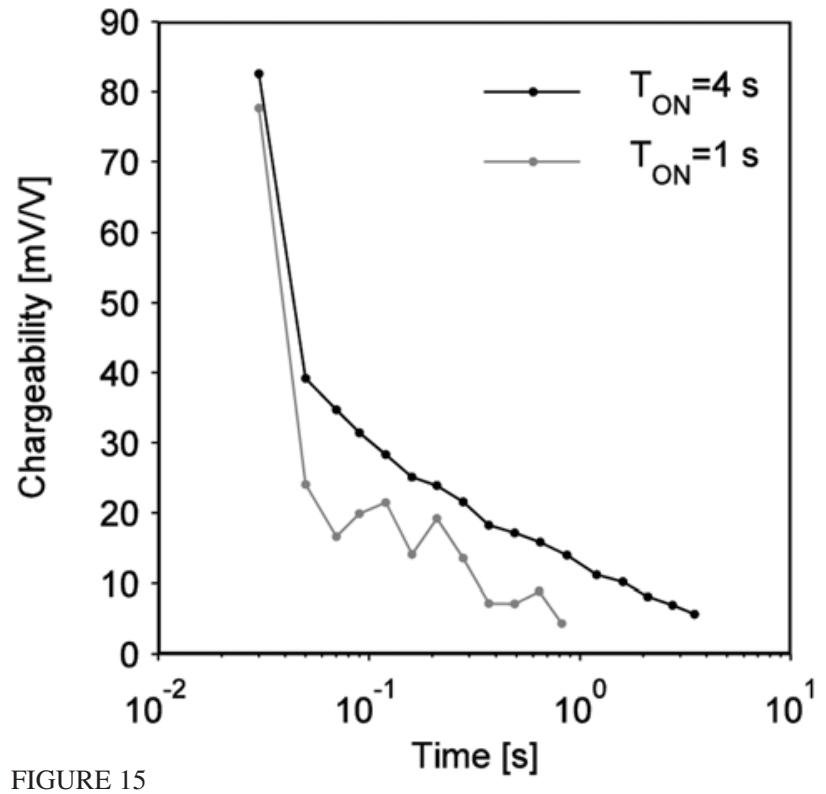

Pulse length and data quality. The grey line is a decay using a $1 \mathrm{~s}$ pulse and the black line is obtained with a $4 \mathrm{~s}$ pulse. The dots mark gate-centre times. The actual decays are indicated in the pseudosection in Fig. 10.

the main aspects taken into account for the choice of the array type and the quadrupole sequence. This study shows that the geometrical factor is another key point that should not be neglected, also depending on the expected field conditions. Three examples 

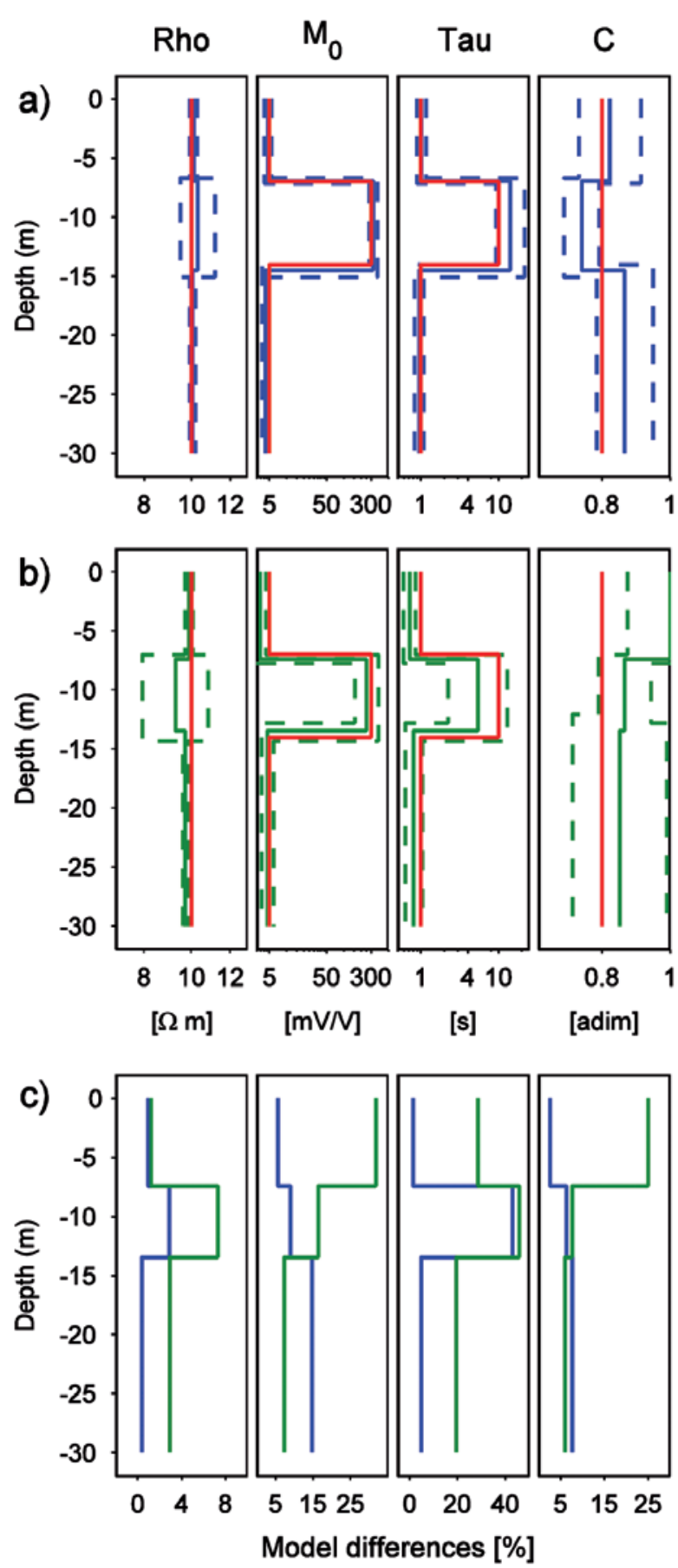

FIGURE 16

Pulse length and information content. a) Results from inversion using a $4 \mathrm{~s} T_{\mathrm{ON}}$. The solid red curve is the true model, the solid blue curve is the inverted model and the dashed curve represents the uncertainty. b) Results from inversion using a $1 \mathrm{~s} T_{\mathrm{ON}}$. The solid red curve is the true model, the solid green line is the inverted model and the dashed green curve represents the uncertainty. c) Percentage of difference between models with respect to initial model for each Cole-Cole parameter (absolute values). Models from a $4 \mathrm{~s}$ pulse are in blue, models from a $1 \mathrm{~s}$ pulse in green. of arrays well-suited for IP acquisition were compared: dipoledipole, gradient and linear grid. The gradient array shows the lower geometrical factor, which helps to reach a higher signal level but linear grid and dipole-dipole are more efficient in avoiding electrode polarization. Furthermore, on the profile presented in this study, the linear grid array shows the best uncertainty analysis. Finally, this study highlights the influence of the duration of current injection on IP data: the longer injection pulse, the larger information content and the higher signal-to-noise ratio.

\section{ACKNOWLEDGEMENT}

This article is an outcome of the EU Interreg IVB project CLIWAT. It has been co-funded by the North Sea Region Programme 2007-2013 under ERDF of the European Union.

This work was supported by the Danish Agency for Science Technology and Innovation funded project RiskPoint - Assessing the risks posed by point source contamination to groundwater and surface water resources under grant number 09-063216.

\section{REFERENCES}

Auken E., Christiansen A.V., Jacobsen B.H., Foged N. and Sørensen K.I. 2005. Piecewise 1D Laterally Constrained Inversion of resistivity data. Geophysical Prospecting 53, 497-506.

Barker R.D. 1989. Depth of investigation of collinear symmetrical fourelectrode arrays. Geophysics 54(8), 1031-1037.

Capizzi P., Cellura D., Cosentino P.L., Fiandaca G., Martorana R., Messina P. et al. 2010. Integrated hydrogeochemical and geophysical surveys for a study of sea-water intrusion. Bollettino di Geofisica Teorica e Applicata 51(4), 285-300.

Capizzi P., Cosentino P.L., Fiandaca G., Martorana R., Messina P. and Vassallo S. 2007. Geophysical investigations at the Himera archealogical site, northern Sicily. Near Surface Geophysics 5(6), 417-426. doi: 10.3997/1873-0604.2007024

Carlson N.R., Hare J. and Zonge K.L. 2001. Buried landfill delineation with induced polarization: Progress and problems. Proceedings of the Symposium of the Application of Geophysics to Engineering and Environmental Problems (SAGEEP), Denver, Colorado.

Christiansen A.V., Auken E. and Sørensen K.I. 2006. The transient electromagnetic method. In: Groundwater Geophysics, a tool for hydrogeology, (ed. R. Kirsch), 179-225. Springer.

Dahlin T., Leroux V. and Nissen J. 2002. Measuring techniques in induced polarization imaging. Journal of Applied Geophysics 50, 279-298.

Dahlin T. and Zhou B. 2002. Gradient and mid-point referred measurements for multi-channel 2D resistivity imaging. Proceedings of the Integrated Case Histories session, 8th meeting EEGS-ES, Aveiro, Portugal, 157-160.

Dahlin T. and Zhou B. 2004. A numerical comparison of 2D resistivity imaging with 10 electrodes arrays. Geophysical Prospecting 52, 379-398.

Dahlin T. and Zhou B. 2006. Multiple-gradient array measurements for multi-channel 2D resistivity imaging. Near Surface Geophysics 4(2), 113-123. doi: 10.3997/1873-0604.2005037

Doherty R., Kulessa B., Ferguson A.S., Larkin M.J., Kulakov L.A. and Kalin R.M. 2010. A microbial fuel cell in contaminated ground delineated by electrical self-potential and normalized induced polarization data. Journal of Geophysical Research-Biogeosciences 115. doi:10.1029/2009JG001131

Effersø F., Auken E. and Sørensen K.I. 1999. Inversion of band-limited TEM responses. Geophysical Prospecting 47, 551-564. 
a)

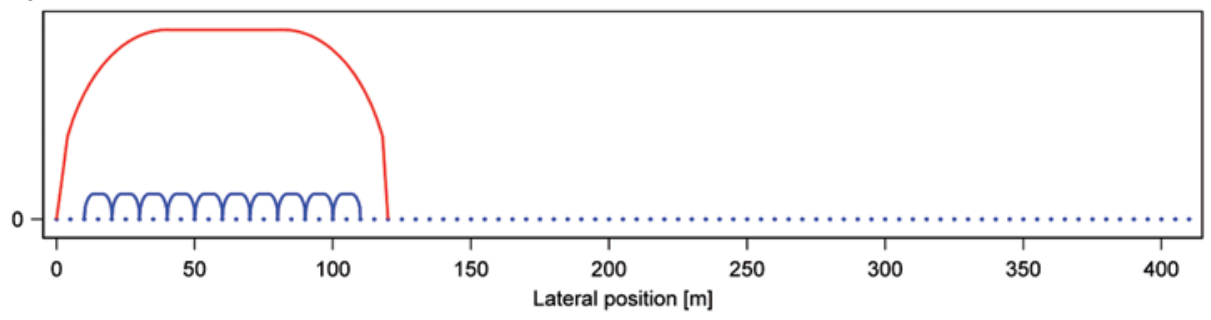

FIGURE 17

Sketch of the different protocols used in this study. a) Gradient array. b) Dipole-dipole. c) Linear grid.

b)

Dipole Dipole

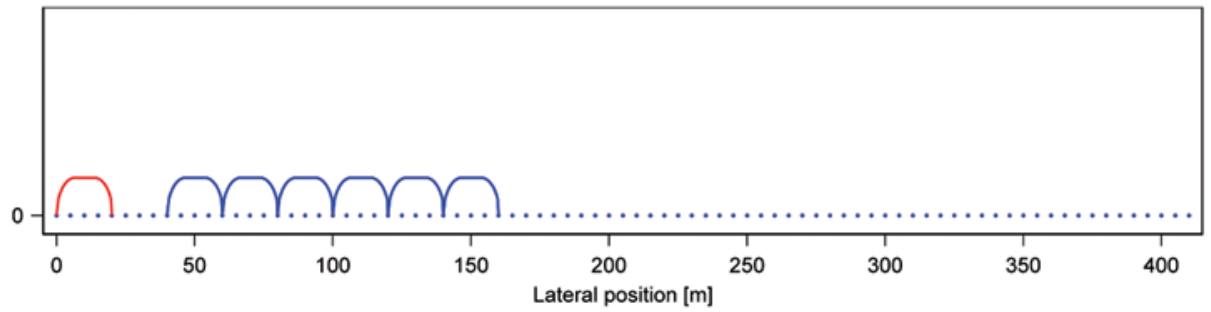

c)

Linear Grid

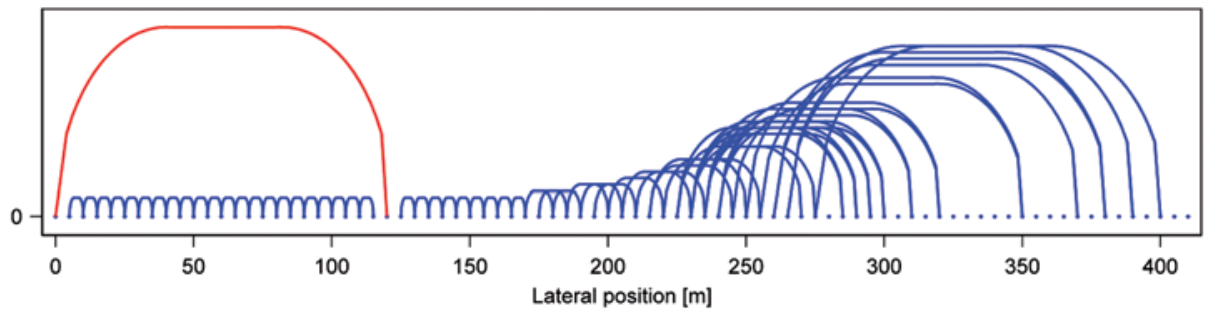

- Injecting current - Measuring potential

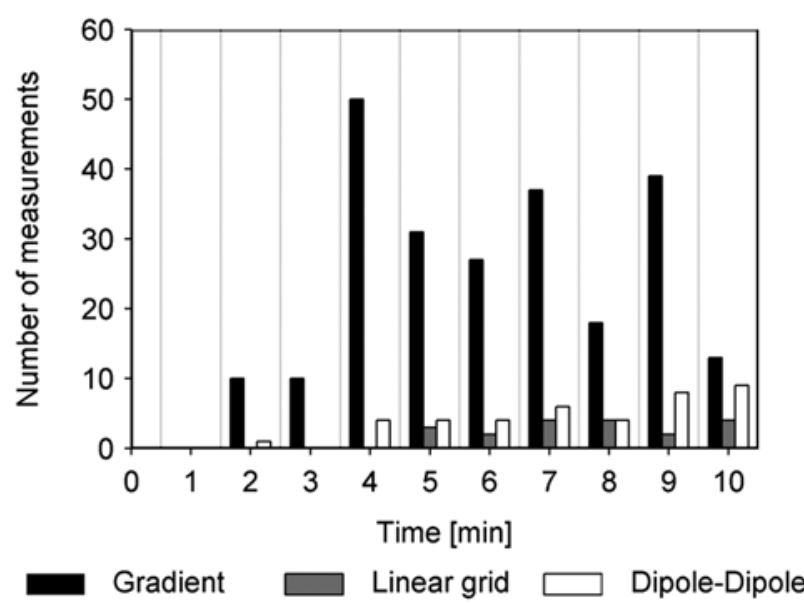

FIGURE 18

Time gap required for current electrodes to be used for measuring potentials. Three different arrays are compared.

Fiandaca G., Auken E., Christiansen A.V. and Gazoty A. 2012. Time Domain Induced Polarization: Full Decay Forward Response Modeling and 1D Laterally Constrained Inversion of Cole-Cole Parameters. Geophysics 77(3), E213-E225. doi: 10.1190/GEO20110217.1
Fiandaca G., Martorana R. and Cosentino P.L. 2005. Use of linear grid array in 2D resistivity tomography in near surface. Near Surface 2005, Palermo, Italy, Expanded Abstracts, A023.

Gazoty A., Fiandaca G., Pedersen J., Auken E. and Christiansen A.V. 2012a. Mapping of landfills using time-domain spectral induced polarization data: the Eskelund case study. Near Surface Geophysics 10(6), 563-574. doi: 10.3997/1873-0604.2012046

Gazoty A., Fiandaca G., Pedersen J., Auken E., Christiansen A.V. and Pedersen J.K. 2012b. Application of time domain induced polarization to the mapping of lithotypes in a landfill site. Hydrology and Earth System Sciences 16, 1-12. doi: 10.5194/hess-16-1-2012

Hördt A., Blaschek R., Kemna A. and Zisser N. 2007. Hydraulic conductivity estimation from induced polarisation data at the field scale - The Krauthausen case history. Journal of Applied Geophysics 62, 33-46.

Hördt A., Hanstein T., Hönig M. and Neubauer F.M. 2006. Efficient spectral IP-modelling in the time domain. Journal of Applied Geophysics 59, 152-161. doi: 10.1016/j.jappgeo.2005.09.003

Johansson B., Jones S., Dahlin T. and Flyhammar P. 2007. Comparisons of 2D- and 3D-Inverted Resistivity Data As Well As of Resistivity- and IP-Surveys on a Landfill. Near Surface 2007, Istanbul, Turkey, Expanded Abstracts, P42.

Johnson I.M. 1984. Spectral induced polarization parameters as determined through time-domain measurements. Geophysics 49, 1993-2003.

Kemna A., Binley A. and Slater L. 2004. Crosshole IP imaging for engineering and environmental applications. Geophysics 69, 97-107. doi: 10.1190/1.1649379

LaBrecque D. and Daily W. 2008. Assessment of measurement errors for galvanic-resistivity electrodes of different composition. Geophysics 73(2), F55-F64. doi: 10.1190/1.2823457 
LaBrecque D.J., Miletto M., Daily W., Ramirez A. and Owen E. 1996. The effects of noise on Occam's inversion of resistivity tomography data. Geophysics 61, 538-548.

Leroux V., Dahlin T. and Svensson M. 2007. Dense resistivity and induced polarization profiling for a landfill restoration project at Härlöv, Southern Sweden. Waste Management \& Research 25, 49-60.

Martorana R., Fiandaca G., Casas Ponsati A. and Cosentino P.L. 2009. Comparative tests on different multi-electrode arrays using models in near-surface geophysics. Journal of Geophysics and Engineering 6, 1-20. doi: 10.1088/1742-2132/6/1/001

Munkholm M.S. and Auken E. 1996. Electromagnetic noise contamination on transient electromagnetic soundings in culturally disturbed environments. Journal of Environmental and Engineering Geophysics 1, 119127.

Pelton W.H., Ward S.H., Hallof P.G., Sill W.R. and Nelson P.H. 1978. Mineral discrimination and removal of inductive coupling with multifrequency IP. Geophysics 43, 588-609. doi: 10.1190/1.1440839

Radic T. 2004. Elimination of cable effects while multi-channel SIP measurements. Near Surface 2004, Utrecht, The Netherlands, Expanded Abstracts, P029.

Ramirez A., Daily W., Binley A. and LaBrecque D. 1999. Electrical Impedance Tomography of Known Targets. Journal of Environmental and Engineering Geophysics 4, 11-26.

Schön H.J. 1996. Physical Properties of Rocks: Fundamentals and Principles of Petrophysics. Pergamon, New York, 583.

Slater L. and Binley A. 2006. Synthetic and field-based electrical imaging of a zerovalent iron barrier: Implications for monitoring long-term barrier performance. Geophysics 71(5), B129-B137.

Slater L.D., Ntarlagiannis D., Day-Lewis F.D., Mwakanyamale K., Versteeg R.J., Ward A. et al. 2010. Use of electrical imaging and distributed temperature sensing methods to characterize surface water-groundwater exchange regulating uranium transport at the Hanford 300 Area, Washington. Water Resources Research 46, W10533, 13. doi:10.1029/2010WR009110

Sogade J.A., Scira-Scappuzzo F., Vichabian Y., Shi W., Rodi W., Lesmes D.P. and Dale Morgan F. 2006. Induced-polarization detection and mapping of contaminant plumes. Geophysics 71(3), B75-B84. doi: $10.1190 / 1.2196873$

Tarantola A. and Valette B. 1982. Generalized nonlinear inverse problems solved using a least squares criterion. Reviews on Geophysics and Space Physics 20, 219-232.

Tombs J.M.C. 1981. The feasibility of making spectral IP measurements in the time domain. Geoexploration 19, 90-102.

Vanhala H. 1997. Mapping oil-contaminated sand and till with the spectral induced polarization (IP) method. Geophysical Prospecting 45, 303-326.

Vanhala H., Soininen H. and Kukkonen I. 1992. Detecting organic chemical contaminants by spectral-induced polarization method in glacial till environment. Geophysics 57, 1014-1017. doi: 10.1190/1.1443312

Vaudelet P., Schmutz M., Pessel M., Franceschi M., Guérin R., Atteia O. et al. 2011. Mapping of contaminant plumes with geoelectrical methods. A case study in urban context. Journal of Applied Geophysics 75, 738-751.

Wilkinson P.B., Loke M.H., Meldrum P. I., Chambers J.E., Kuras O., Gunn D.A. and Ogilvy R.D. 2012. Practical aspects of applied optimized survey design for electrical resistivity tomography. Geophysical Journal International 189, 428-440. doi: 10.1111/j.1365-246X.2012.05372.x

Williams K.H., Kemna A., Wilkins M.J., Druhan J., Arntzen E., N'Guessan A.L. et al. 2009. Geophysical monitoring of coupled microbial and geochemical processes during stimulated subsurface bioremediation. Environmental Science and Technology 43, 6717-6723.

Yuval and Oldenberg D.W. 1997. Computation of Cole-Cole parameters from IP data. Geophysics 62, 436-448. doi: 10.1190/1.1444154

Zonge K.L. and Hughes L.J. 1985. The effect of electrode contact resistance on electric field measurements. Proceedings of the 55th SEG Convention, Washington, USA, 231-234.

\section{APPENDIX A1}

\section{Gradient array}

The gradient array (Dahlin and Zhou 2006), also known as the multiple gradient, is carried out by injecting a current between electrodes with a separation of $a^{*}(n+2)$ and measuring the potential differences between all the electrode couples with spacing $a$ comprised within the current dipole, $n$ being the number of potential readings for a current injection (Fig. 17a). The same layout is applied by sweeping the profile with different spacing $a$. The design of such an array allows maximizing the time interval before a current injection electrode is used for potential measurements, by a proper sorting of the quadrupole sequence. The sequence used for the present study was constructed by using $n=10$ and $a=1,2$, 4 and 6 electrode spacing over 83 electrodes. The quadrupoles with $a=1$ electrode spacing were swept, scanning for each electrode, while the others ( $a=2,4$ and 6 electrode spacing) scanning for every two electrodes, for a total amount of 1250 quadrupoles.

\section{APPENDIX A2 \\ Dipole-dipole}

In this layout, the dipole used for the potential measurements is outside the dipole used for current injection but both have the same length $a$ (Fig. 17b). The distance between the two dipoles, also called the dipole separation factor, is usually an integer multiple $n$ of the dipole. The sequence used for the present study was constructed by using $a=1,4,6$ and 10 electrode spacings and $n=10$, over 83 electrodes (but excluding the quadrupoles with a geometrical factor greater than $21100 \mathrm{~m}$ ). The quadrupoles with $a=1$ electrode spacing were swept scanning for each electrode, while the others ( $a=4,6$ and 10 electrode spacing) scanning for every two electrodes, for a total amount of 1154 quadrupoles.

\section{APPENDIX A3}

\section{Linear grid}

The linear grid array (Fiandaca et al. 2005; Capizzi et al. 2007; Martorana et al. 2009; Capizzi et al. 2010) was designed in order to minimize the number of current injections and hence the number of electrodes used for current injection. Still, a resolution comparable to more common arrays such as the Wenner array or the dipole-dipole array is maintained. For each current dipole of length $a$, potential measurements are carried out for every other adjacent pair of electrodes, both inside and outside the current dipole (Fig. 17c). A threshold can be selected for the upper value of the geometrical factor in the quadrupole sequence. In this case, the length of potential dipoles in the protocol design is increased to maintain the geometrical factor of the corresponding quadrupole below the threshold.

This array has the advantage of using a limited number of current dipoles compared to a large number of potential dipoles, thereby minimizing electrode polarization. In order to fully use the array benefits, in terms of acquisition time and regarding the electrode polarization effect, a high number of channels in the acquisition system is preferable. 
In the sequence used for the present study, 11 out of 83 electrodes were used to send out current. 24 different current dipoles were used, scanning for every 8 electrodes, with length $a=8,24$, 64,72 and 80 electrode spacing, for a total amount of 1194 quadrupoles. A threshold equal to $3600 \mathrm{~m}$ was used for the geometrical factor.

\section{APPENDIX A4}

\section{Electrode polarization}

The electrode polarization effect can have two origins (Dahlin et al. 2002):

1) It can be a strong residual potential because the potential electrodes have previously been used to transmit current. In this case, it can take several tens of minutes for the effect to vanish.

or

2) It can be a charge-up effect occurring at the contact between the soil and the electrode, depending on electrochemical phenomena. This effect is most severe just after inserting the electrode into the soil.

There are several ways of reducing the electrode polarization effect. One way is to use non- polarizable electrodes (Carlson et al. 2001), since the potential difference due to the charge-up is much smaller and relatively stable (Dahlin et al. 2002). Another way is the use of an appropriate array that can either limit the use of electrodes for current injection (e.g., the linear grid, see Appendix A3) or maximize the time between an electrode is used for current injection until the same electrode is used for potential measurements (e.g., the gradient array, see Appendix A1).

A comparison based on a TDIP acquisition was performed using the linear grid, the gradient and the dipole-dipole, on the same profile. For each array, the time between an electrode is used for sending out current and making a potential measurement was calculated. The results are shown in Fig. 18. For short time gaps, there is a significant difference between the three protocols: the gradient array involves a bigger number of measurements within a time-lapse of five minutes, whereas the linear grid allows a longer gap before the injecting electrode is used for measuring potential. The electrode polarization effect is believed to vanish after tens of minutes (Dahlin et al. 2002), so if a measurement is performed within this time range, it might be affected. All the arrays used in this study involve a time gap smaller than the necessary time to neglect the electrode polarization effect. However the linear grid, closely followed by the dipole-dipole, shows a better efficiency in this respect.

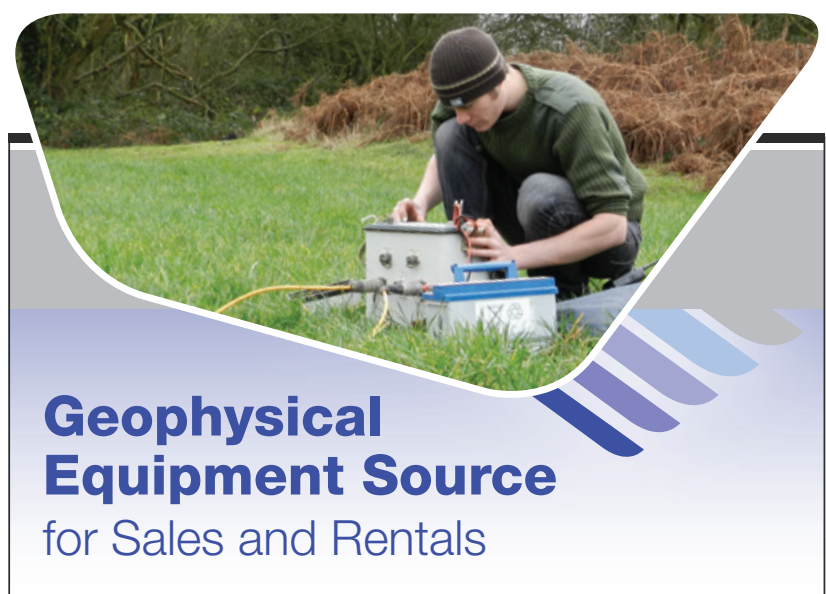

Our expertise covers mineral exploration, all types of near surface surveys, marine geophysics, UXO and surveys for other manmade hazards

\section{T: +44 (0)1525 $383438 \quad$ E: sales@geomatrix.co.uk}

20 Eden Way,

Pages Industrial Park, Leighton Buzzard, Beds LU7 4TZ. UK.

\section{Geomatrix} EARTH SCIENCE LTD 